\title{
NFC Ticketing Mobile Service
}

\author{
${ }^{1}$ Mrs. Geeta S. Kavitake, ${ }^{2}$ Kanchan S. Gorde \\ ${ }^{1,2}$ Electronics Department, Terna college of engg. / Mumbai university, India
}

\begin{abstract}
: near field communication is young radio technology which operates within $10 \mathrm{~cm}$ and data rates available are 106, 212, $424 \mathrm{kbps}$ and mobile ticketing is considered as one of the most promising application of $n f c$. customer could use their mobile phone which is configured with rfid tag. furthermore $n f c$ enabled unit have been placed at station in the area containing station specific information. by placing the mobile phone in front of rfid reader one can get the ticket on mobile phone. nfc ticketing has many advantages over smart card application such as it is faster in speed.

Keywords: communication mode mobile, $n f c$, system structure, ticketing
\end{abstract}

\section{INTRODUCTION}

Over the last few years, the growing availability of wireless technologies such as Wi-Fi, Bluetooth, Radio Frequency Identification (RFID), made users increasingly more "mobile", setting them free from a fixed working station, so allowing them to perform day-to-day actions anytime and everywhere. Each one of these technologies is suitable for a particular context of use within a specific scenario [1]. For example, Bluetooth is particularly useful when the device the user wants to interact with, is not on his/her line of sight. However, a Bluetooth-based interaction is not immediately perceivable for the user because it is not possible to associate its operation mode (scanning the surroundings), to an action occurring in the real life. Conversely, by means of an RFID-based interaction, the user can replicate the act of "touching" an object to interact with it in the same way that people reach a switch to light a room, or press a button, to move the elevator towards a given floor, considerably decreasing the user's cognitive load.

In such a scenario, defined by a growing wireless infrastructure and by the mobility of users, it is hard to imagine a device that has more influence on the people's life and is, at the same time, so universally accepted, as the mobile phone. For this reason, wireless technologies for mobiles, based on RFID technology, such as Near Field Communication (NFC) and Felicity Card (FeliCa®, a contactless technology developed by Sony Corporation), seem to be the more suitable human-computer interaction solution, because they inherit advantages from both RFID and mobile technologies. As for cell phones, these are not only largely used by people all over the world; they are also personal objects, always carried in pockets, or bags, by their owners. On the other hand, RFID services are very easy to use and they can rely on a constantly growing infrastructure. Examples are electronic ticketing systems in use in London, Milan, Rome, all based on contactless cards.

A key application of Near Field Communication (NFC) can be found in the field of Electronic Fare Management.The integration of NFC in the field of public transport has already been analysed in a few prior trials and research projects. In 2005 the German transport association RMV (Rhein-Main-Verkehrsverbund) started a pilot project, where customers could use their NFC enabled mobile phone to purchase tickets. Based on a best-price-policy passengers only had to check in/out at a terminal in the bus when they entered or left, in order to receive the cheapest ticket for the route [2]. Furthermore, RFID tags (called Con Tags) have been placed at many stations in the area containing station specific information. Touching one of these tags opens the RMV mobile portal on the mobile phone and also fills out the departure station information with the data read from the tag [3].

In this project the main aim is to introduce a new ticketing system for the local train system. This system will make use of the Near Field Communication technology along with the mobile phone (Java and GPRS Enabled) to carry out the ticketing transactions.

\section{BACKGROUND}

In this section we describe the evolution of the ticket notion. A ticket is a little paper certificate, which represents the performed payment to access several services (cinemas, theaters or other kind of services as public transportation): a ticket is a contractual tool for service delivery. Over the last years, thanks to the increasing use of computers, there was a gradual transition towards the disappearance of "paper" in favor of digital documents.

This process did not spare the "ticket": an electronic ticket, or e-ticket, was originally used to represent the purchase of a seat on a passenger airline, usually through a website or by phone. To check-in by means of an e-ticket, the passenger usually comes to the check-in counter and presents the confirmation or reservation code. 
This form of airline ticket rapidly replaced the old paper tickets and became mandatory for IATA members to accept an e-ticket as of June 1st, 2008.

Afterwards, electronic tickets were introduced in road, urban or rail public transportation as well. For example from January 2006, the East Japan Railway Company (JR East), NTT DoCoMo, Inc. and Sony corporation (Sony) offer a service combining DoCoMo's i-mode ${ }^{\circledR}$ FeliCa ${ }^{\circledR}$ smart-card handset and Suica, JR East's IC card train ticket. The Mobile Suica service enables i-mode ${ }^{\circledR}$ FeliCa ${ }^{\circledR}$ handsets to be used as Suica cards. Mobile Suica customers, in addition to enjoying all current Suica features, are able to recharge stored fares and purchase commuter passes anytime and anywhere and also check the balance of stored fare usage on the screen of the handsets. Another example of an e-ticket is given by the Czech bus reservation system AMSBUS, which, in February 2007, introduced the product "ejízdenka" on several bus routes. Since November 2007 it has been possible to buy an SMS-ticket (SMS jízdenka) for use on Prague's urban transportation.

In 2006 the Roman public transportation company ATAC introduced the use of mTickets via SMS [4]. In order to access the service, the user had to perform a subscription to his mobile network operator; next the ser has only to send a text message (with a particular syntax) to the service center. The network operator will reply with another text message indicating the ticket type, validity time and other related information. This system has some weak spots, especially regarding the cognitive load of the user: some people, in fact, have some difficulty in drafting an SMS and must also have the burden of remembering the syntax of the text itself; moreover there are problems concerning security issues.

The NFC technology can be a valid aid to overcome these problems thanks to its ease of use and to its higher security level. The first NFC trials "on the field" started in 2004, with a number of leading mobile and set manufacturers, such as Nokia, Motorola and Samsung. The first commercial implementation of NFC products came in 2005 in Hanau (Germany), after a successful ten-month field trial. With their NFC-equipped Nokia 220 mobile phone, citizens of Hanau are now able to purchase and validate public transport tickets [5]

\section{NFC}

A number of major companies, including Nokia, Philips and Sony formed an alliance in 2004 to advance the use of RFID technology in consumer applications. The alliance, the Near Field Communication forum (NFC), was later joined by others such as Visa, MasterCard, Samsung, Microsoft and Motorola. The applications targeted were contactless payment, ticketing, easy information access, and peer-to-peer communication. The NFC concept is based on integrating existing RFID technology, especially ISO 14443based Mifare (Philips) and Felica (Sony), to portable consumer devices, such as mobile phones. The project relies on NFC/RFID technology and the touch me paradigm.

NFC allows a device, usually a mobile phone, to collect data from another device or NFC tag at close range. In many ways, it's like a contactless payment card that is integrated into a phone. In other ways, it's similar to Bluetooth, except that instead of programming two devices to work together, they can simply touch to establish a connection. Near field communication or NFC is short-range wireless technology, operating at a distance of $10 \mathrm{~cm}$ or less. NFC operates at $13.56 \mathrm{MHz}$ with data rate ranging from106 Kbit/s to $48 \mathrm{Kbit} / \mathrm{s}$. Most of the RF energy is concentrated in the allowed $14 \mathrm{kHz}$ bandwidth range, but the full spectral envelope may be as wide as $1.8 \mathrm{MHz}$ when using ASK modulation.

NFC enables two way communications between electronic devices. And has the capability to write to the RFID (Radio Frequency Identification) chip. Therefore bidirectional communication between NFC-equipped cell phone and NFC reader can be established. That makes the possibility to develop complex applications like payment,

Secure exchange of data and identity's authentication [6]. NFC implements touching paradigm. Touching is a famous and interactive method in human lives. This makes NFC technology easy to learn and use. This touching paradigm was initially used in RFID (Radio Frequency Identification) technology. In RFID technology items marked with tags contain transponders which emit messages in the form of signals. RFID readers were used to read those messages. NFC is now integrated with this RFID technology.

The tags to be readable by NFC reader should have 4 to 10 byte unique ID. This unique ID is used for the identification of the tag. There are multiple manufacturers in the industry, so ID's length may vary in size [7]. NFC equipped devices normally operate in three different modes i.e. Card emulation mode, peer-to-peer mode, and reader-writer mode. In card emulation mode NFC device behaves like a reader e.g. NFC tag. This tag has the capacity to store data securely and the applications of this mode are electronic ticketing and payments. In peer to peer mode two devices equipped with NFC can exchange data directly by touching each other. Applications of P2P mode are transferring data between laptop and cell phone like Printing of data by touching laptop with printer. In reader writer mode NFC device can read or write the tags in similar fashion like RFID tags [8].

The application, named NFC Ticketing is being developed following a user-centered approach, so obtaining a balance between the information reductions required by the user and the increase of application 
flexibility. In this way, the NFC Ticketing service will not only be less complex and therefore less expensive (keeping low both the cost of development and implementation) but will also be more usable for a broad community of users. The NFC Ticketing application combines latest-generation technologies (such as NFC) with Radio Frequency Identification (RFID). All the architectural components of the application are fully described, detailing both software and hardware features and also the relative communication scheme.

\subsection{NFC modes of operation:}

RF signal transmission between transmitter and receiver creates the main distinction between NFC and other RF wireless communication modes. NFC depends upon straight magnetic/electrostatic coupling between devices instead of freely broadcasting of radio waves, such as in Wi-Fi. NFC devices can operate on low electric or magnetic field strengths due to its short range communication property [8].

NFC system can operate either in active or passive mode depending upon requirements.

ECMA-340 is the standard which defines the modes of operation.

Table 1: ECMA-340 showing Magnetic

\begin{tabular}{l|r|c|} 
Field level & Field strength & Description \\
\hline $\mathrm{H}_{\text {threshold }}$ & $0.1875 \mathrm{~A} / \mathrm{m}$ & Minimum field detection level \\
\hline $\mathrm{H}_{\min }$ & $1.5 \mathrm{~A} / \mathrm{m} \mathrm{rms}$ & Minimum un-modulated field strength \\
\hline $\mathrm{H}_{\max }$ & $7.5 \mathrm{~A} / \mathrm{rn} \mathrm{rms}$ & Maximum un-modulated field strength \\
\hline
\end{tabular}

\subsubsection{Active Mode}

In this mode both devices generate RF (radio frequency) field to transfer data. In this situation any of the devices can be the initiator and other will be the target. In this situation initiator of the communication will generate field and target will use load modulation. During the communication, the initiator starts the communication in a particular mode at a specific speed. Target finds out the current speed and replies back to the initiator. Termination of the communication takes place either when two devices move out of the range or application gives command to terminate it [9].During communication either initiator or target generates RF field of level $\mathrm{H}$ min that does not go beyond the field level of $\mathrm{H} \max [10]$.

\subsubsection{Passive Mode}

This mode has a key benefit for battery powered devices. For battery powered devices low consumption of battery is the basic priority. Thus NFC allows battery powered devices such as cell phones to operate in passive mode. In this mode RF field is generated on the other side. Thus battery power is saved that was needed to be used for generating RF field. In passive mode target operates continuously between $\mathrm{H}$ min and $\mathrm{H}$ max magnetic field strength [9]. NFC protocol is also compatible with connectionless smart card protocols like Felicia and Mifare. NFC device can work with both smart card and smart card reader. Another benefit of the NFC device is that it can be used as smart card, as well as smart card reader [10].

Devices cannot change mode of communication (Active/Passive) during single transaction unless target is removed or deactivated. Even transfer speed of target to initiator and vice versa may not affect the change in mode [9].

\section{SYSTEM ARCHITECTURE}

The Fig. shows the architecture of the system. And all the required components are explained.

\subsection{Mobile Phone:}

The first component will be a GPRS enabled mobile phone with an RFID chip attached to it. The mobile phone will be fed with a computer program coded entirely in J2ME.

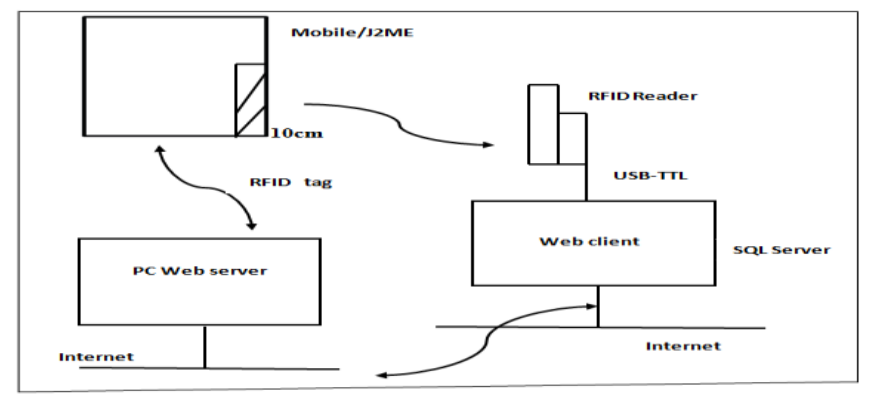

Figure 1: System Architecture

\subsection{RFID Reader:}


The RFID Reader is the second component which is meant to be mounted on the wall of Railway Stations that would be accessible to the commuters. RFID reader modules are also called as interrogators. They convert radio waves returned from the RFID tag into a form that can be passed on to Controllers, which can make use of it. RFID tags and readers have to be tuned to the same frequency in order to communicate. RFID systems use many different frequencies, but the most common and widely used \& supported by our EM18 RFID Reader is $125 \mathrm{KHz}$.

\subsection{Server:}

This is the most important component that is supposed to manage the entire system. The server will consist of the user database i.e. their unique ID's and a computer program coded in VB.NET. The server consists of three tables' viz. Account, Fare, Ticket. Account table consists of information related to customer such as CID (customer ID), password, balance and RFID tag number. Fare table consists of fares to be charged with respect station and class. And ticket consists of ticket related information such as TID (ticket ID), src (source), and des (destination).

\subsection{USB to TTL converter:}

The CP2102 is a highly-integrated USB-to-UART Bridge controller providing a simple solution for updating RS-232 designs to USB using a minimum of components and PCB space. The CP2102 includes a USB 2.0 full-speed function controller, USB transceiver, oscillator, EEPROM, and asynchronous serial data bus (UART) with full modem control signals in a compact $5 \times 5 \mathrm{~mm}$ MLP-28 package. No other external USB components are required.

The on-chip EEPROM may be used to customize the USB Vendor ID, Product ID, Product Description String, Power Descriptor, Device Release Number, and Device Serial Number as desired for OEM applications. The EEPROM is programmed on-board via the USB allowing the programming step to be easily integrated into the product manufacturing and testing process.

The various sections of USB to TTL CP2102 are discussed here.

\subsubsection{USB Function controller and Transceiver:}

The Universal Serial Bus function controller in the CP2102 is a USB 2.0 compliant full-speed device with integrated transceiver and on-chip matching and pull-up resistors. The USB function controller manages all data transfers between the USB and the UART as well as command requests generated by the USB host controller and commands for controlling the function of the UART.

The USB Suspend and Resume signals are supported for power management of both the CP2102 device as well as external circuitry. The CP2102 will enter Suspend mode when Suspend signaling is detected on the bus. On entering Suspend mode, the CP2102 asserts the SUSPEND and SUSPEND signals. SUSPEND and SUSPEND are also asserted after a CP2102 reset until device configuration during USB Enumeration is complete.

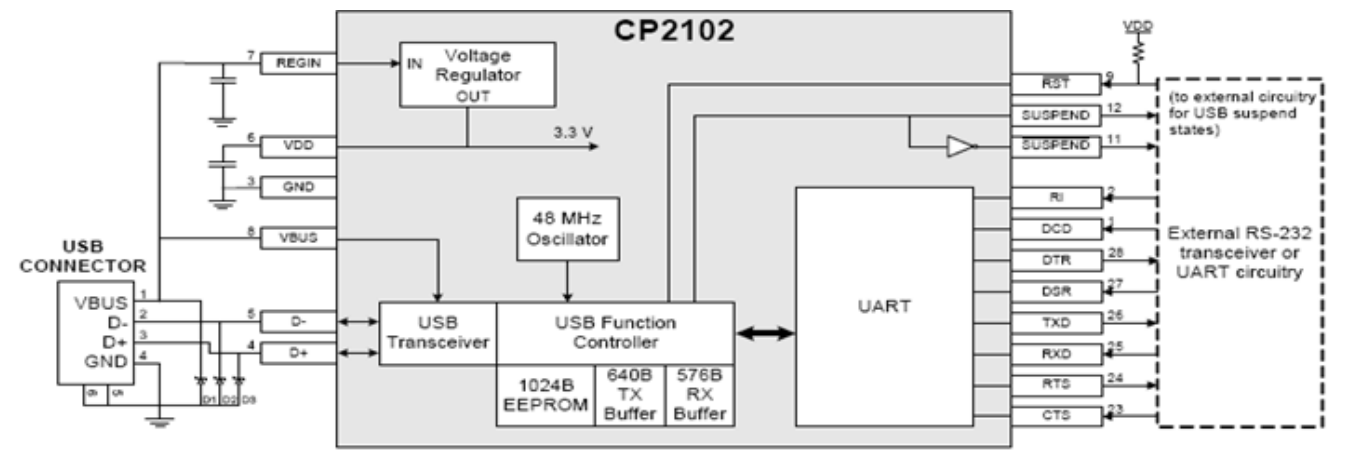

Figure 2: USB to TTL CP2102

\subsubsection{UART Interface:}

The CP2102 UART interface consists of the TX (transmit) and RX (receive) data signals as well as the RTS, CTS, DSR, DTR, DCD, and RI control signals. The UART supports RTS/CTS, DSR/DTR, and X-On/XOff handshaking. The UART is programmable to support a variety of data formats and baud rates. The data format and baud rate programmed into the UART is set during COM port configuration on the PC. The data formats and baud rates available are listed in Table 2.

Table 2: Data Formats and Baud Rates 


\begin{tabular}{|l|l|}
\hline Stop bits & $1,1.5,2$ \\
\hline Parity type & None , even, odd, mark, space \\
\hline Baud Rate & $300,600,1200,1800,2400,4000,4800$, \\
& $7200,9600,14400,16000,19200,28800$, \\
& $38400,51200,56000,57600,64000$, \\
& $76800,15200,128000,153600,230400$, \\
& $250000,256000,460800,500000,576000$, \\
& 921600 \\
\hline
\end{tabular}

\subsubsection{Voltage Regulator:}

The CP2102 includes an on-chip 5 to $3 \mathrm{~V}$ voltage regulator. This allows the CP2102 to be configured as either a USB bus-powered device or a USB self-powered device. When enabled, the $3 \mathrm{~V}$ voltage regulator output appears on the VDD pin and can be used to power external $3 \mathrm{~V}$ devices. Alternatively, if $3 \mathrm{~V}$ power is supplied to the VDD pin, the CP2102 can function as a USB self-powered device with the voltage regulator disabled. For this configuration, it is recommended that the REGIN input be tied to the $3 \mathrm{~V}$ net to disable the voltage regulator.

\subsubsection{Internal EEPROM}

The CP2102 includes an internal EEPROM that may be used to customize the USB Vendor ID, Product ID, Product Description String, Power Descriptor, Device Release Number, and Device Serial Number as desired for OEM applications. Customization of the USB configuration data is optional. If the EEPROM is not programmed with OEM data, the default configuration data shown in Table 3 is used. However, a unique serial number is required for OEM applications in which it is possible for multiple CP2102-based devices to be connected to the same PC.

Table 3. Default USB Configuration Data

\begin{tabular}{|c|c|}
\hline Name & Value \\
\hline Vendor ID & 10C4h \\
\hline Product ID & EA60h \\
\hline $\begin{array}{c}\text { Power Descriptor } \\
\text { (Attr.) }\end{array}$ & $80 \mathrm{~h}$ \\
\hline $\begin{array}{c}\text { Power } \\
\text { Descriptor(Max. } \\
\text { power) }\end{array}$ & $32 \mathrm{~h}$ \\
\hline Release Number & 0100h \\
\hline Serial Number & 0001 (63 characters maximum) \\
\hline $\begin{array}{c}\text { Product Description } \\
\text { String }\end{array}$ & $\begin{array}{c}\text { "CP2102 USB to UART Bridge Controller" } \\
\text { (126 characters maximum) }\end{array}$ \\
\hline
\end{tabular}

\subsection{RFID 125Khz Tag:}

This is a basic RFID Tag Card used for presence sensing, Access Control etc. Works in the $125 \mathrm{kHz}$ RF range. These tags come with a unique 32-bit ID and are not re-programmable. It is EM4001 ISO based RFID IC, consists of $125 \mathrm{kHz}$ carrier and supports 2kbps ASK and Manchester encoding. The 64-bit data stream consists of Header +ID + Data + parity.

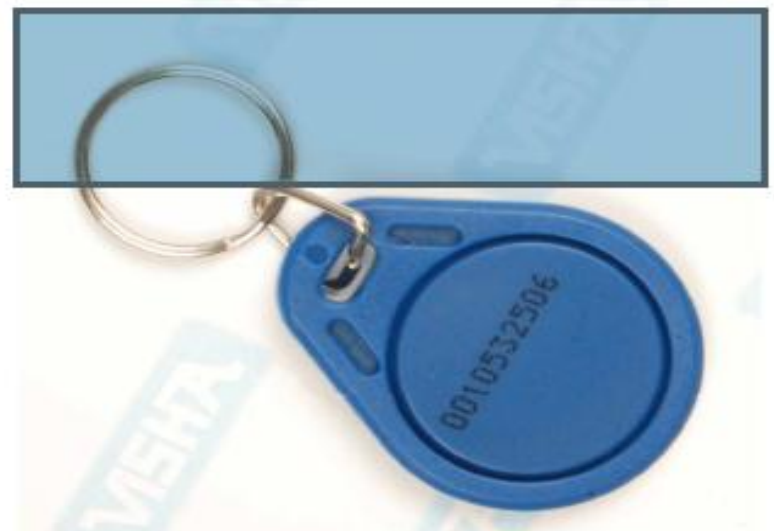

Figure 3: EM4001 RFID Tag 


\section{WORKING}

Initially the user willing to issue a ticket will open the application fed into his mobile phone. The application would consist of all the necessary details to be entered for issuing a basic ticket like destination, no of consumers, etc, after successfully entering the details the user will submit those over the internet.

The next step would be that the user will have to quickly get his mobile phone scanned with the RFID Reader mounted on the Railway Stations, which will lead scan the RFID tag on the users' phone thus obtaining his unique ID. This ID will be transferred over to the Server. The Server in turn will connect to the internet to fetch the details entered by the user and create a ticket in RMS (record management store) format. RMS format is a kind of data storage format which cannot be tampered by the user. This RMS ticket will be sent to the users' mobile phone via the internet thus completing the entire transaction. The ticket will be a timed version which will last for a stipulated time in which the user is meant to complete his journey.

In the meanwhile the server maintains an account of every user and while a ticket or transaction has been requested the amount will be deducted from the balance, further making it easier in the payment terms too. This project consists of software as well as hardware components that need to go together for proper working of both. The implementation of this project began with the obvious first step i.e. collecting all the required details to kick start the project viz. RFID Model features, NFC Concept.

The next step is studying the concepts of programming required for the project viz. Serial Port controlling, Database creation and connectivity using VB.NET. MIDlet creation in J2ME for mobile phone application which acts as RFID tag and GPRS connectivity. Integration of all the above components and communicating Mobile phone and computer applications is required. Mounting up of RFID reader and USB to TTL creating a representation of the NFC Model.

\section{CONCLUSION}

Mobile and web services market is growing rapidly and services are helping users to make their lives easy. As mobile is battery powered device it will help to grow the market of nfc. In this review; an example of the NFC ticketing application has been discussed. Mobile ticketing will play an important role in introducing users to the notion of using a mobile phone for mobile commerce. There might be creative applications and services being introduced that allow consumers to buy and store tickets on their mobile phone. This will become very convenient for consumer. Other than ticketing it nfc mobile can also be used for contactless payment, easy information access, and peer to peer communication.

NFC suffers from security issue.NFC by itself cannot provide protection against eavesdropping or data modifications. The only solution to achieve this is the establishment of a secure channel over NFC. This can be done very easily, because the NFC link is not susceptible to the Man-in-the-Middle attack. Therefore, well known and easy to apply key agreement techniques without authentication can be used to provide a standard secure channel. This resistance against Man-in-the-Middle attacks makes NFC an ideal method for secure pairing of devices.

\section{Examples follow:}

\section{REFERENCES}

\section{Journal Papers:}

[1] E. Rukzio, G. Broll, K. Leichtenstern, A. Schmidt, "Mobile Interaction with the Real World: An Evaluation and Comparison of Physical Mobile Interaction Techniques," in Proc. AmI-07 European Conference on Ambient Intelligence, 2007, Darmstadt, B. Schiele et al. (Eds.), Springer, pp. 1-18, Lecture Notes in Computer Science 4794.

[2] J. Langer and M. Roland, Anwendungen und Technik von Near Field Communication (NFC), 1st ed. Springer, 2010.

[3] "RMV," Rhein-Main-Verkehrsverbund, retrieved on Oct. 20th, 2011. [Online]. Available: http://www.rmv.de/en/Fahrplanauskunft/Fahrplaene/Fahrplaene fuer Handy und Co/39706/HandyPortal.html

[4] Moroni A., M-PAyments: La Pubblica Amministrazione passa per il telefonino (Trad. En. M-Payments: Italian Public Aministration goes through mobile phone). Degree Thesis in Human Computer Interaction, 2007, University of Rome "Sapienza", Faculty of Communication Science.

[5] U. Biader Ceipidor, C.M. Medaglia, A. Moroni, G. Orlandi, S. Sposato, "NFC: Integration between RFID and Mobile, state of the art and future developments," in Proc. RFIDays, 2008, Rome, Gaetano Marrocco Editor, pp. 76-79, Internal Report RR-08-69.

[6] Eamonn O’Neill, Peter Thompson, Stavros Garzonis, and Andrew Warr," Reach Out and Touch: Using NFC and 2D Barcodes for Service Discovery and Interaction with Mobile Devices", UK, 2007

[7] ECMA-340 Standard, Near Field Communication Interface and Protocol (NFCIP-1), 2nd edition, December 2004

[8] GiulianoBeneli and Alessandro Pozzebon,"Near Field communication and Health: Turning a mobile phone into an interactive Multipurpose Assistant in health care scenarios", Italy, 2010

[9] Near Field Communication, White paper, ECMA international, December 2003

[10] Renee Montes,"Examining the technology, security and application of NFC and Evaluateing the possible success of near field communication application in US Markets", Master thesis, Bowie State University, May 2009 